EPJ Web of Conferences 116, 11007 (2016)

DOI: $10.1051 /$ epjconf/201611611007

(C) Owned by the authors, published by EDP Sciences, 2016

\title{
Global $3 v$ oscillation analysis: Status of unknown parameters and future systematic challenges for ORCA and PINGU
}

\author{
Francesco Capozzi ${ }^{1,2}$, Eligio Lisi ${ }^{2, a}$, and Antonio Marrone ${ }^{1,2}$ \\ ${ }^{1}$ Dipartimento Interateneo di Fisica, Università di Bari, via Amendola 173, 70126 Bari, Italy \\ ${ }^{2}$ Istituto Nazionale di Fisica Nucleare, Sezione di Bari, via Orabona 4, 70126 Bari, Italy
}

\begin{abstract}
Within the standard $3 v$ oscillation framework, we illustrate the status of currently unknown oscillation parameters: the $\theta_{23}$ octant, the mass hierarchy (normal or inverted), and the possible CP-violating phase $\delta$, as derived by a (preliminary) global analysis of oscillation data available in 2015 . We then discuss some challenges that will be faced by future, high-statistics analyses of spectral data, starting with one-dimensional energy spectra in reactor experiments, and concluding with two-dimensional energy-angle spectra in large-volume atmospheric experiments. It is shown that systematic uncertainties in the spectral shapes can noticeably affect the prospective sensitivities to unknown oscillation parameters, in particular to the mass hierarchy.
\end{abstract}

\section{Introduction}

The 2015 Nobel Prize in Physics, awarded jointly to Takaaki Kajita and Arthur B. McDonald "for the discovery of neutrino oscillations, which shows that neutrinos have mass" [1], represents both the crowning point of a long quest for neutrino flavor transformations, and an incitement to further proceed in understanding the physics of $v$ masses and mixings. Indeed, world neutrino data have established a simple $3 v$ framework, where the three known flavor states $v_{\alpha}(\alpha=e, \mu, \tau)$ are mixed with three mass states $v_{i}(i=1,2,3)$, separated by two squared mass gaps $\delta m^{2}$ and $\Delta m^{2}$, via three mixing angles $\theta_{12}, \theta_{13}, \theta_{23}$ and a possible CP-violating phase $\delta$ [2]. Although global $v$ data analyses such as [3] provide precise constraints on five parameters (the squared mass gaps and the three mixing angles), we still do not know the octant of $\theta_{23}(<\pi / 4$ or $>\pi / 4)$, the sign of $\Delta m^{2}$ which tells the mass spectrum hierarchy (normal NH for $+\Delta m^{2}$ or inverted IH for $-\Delta m^{2}$ ), and the value of the Dirac phase $\delta$ that could induce CP violating effects in the leptonic sector (if $\delta \neq 0, \pi$ ). Further unknowns, not considered herein, include the absolute $v$ mass scale and the Dirac or Majorana nature of the neutrino fields [2], as well as possible new neutrino states or interactions beyond the standard $3 v$ framework.

In this contribution we illustrate the status of the unknown parameters $\theta_{23}-\pi / 4, \operatorname{sign}\left(\Delta m^{2}\right)$, and $\delta$, in the light of a recent global neutrino data analysis. We then argue that future analyses of highstatistics (and large volume) neutrino experiments will face new and unprecedented challenges, related

\footnotetext{
ae-mail: eligio.lisi@ba.infn.it
}

This is an Open Access article distributed under the terms of the Creative Commons Attribution License 4.0, which permits unrestricted use, distribution, and reproduction in any medium, provided the original work is properly cited. 


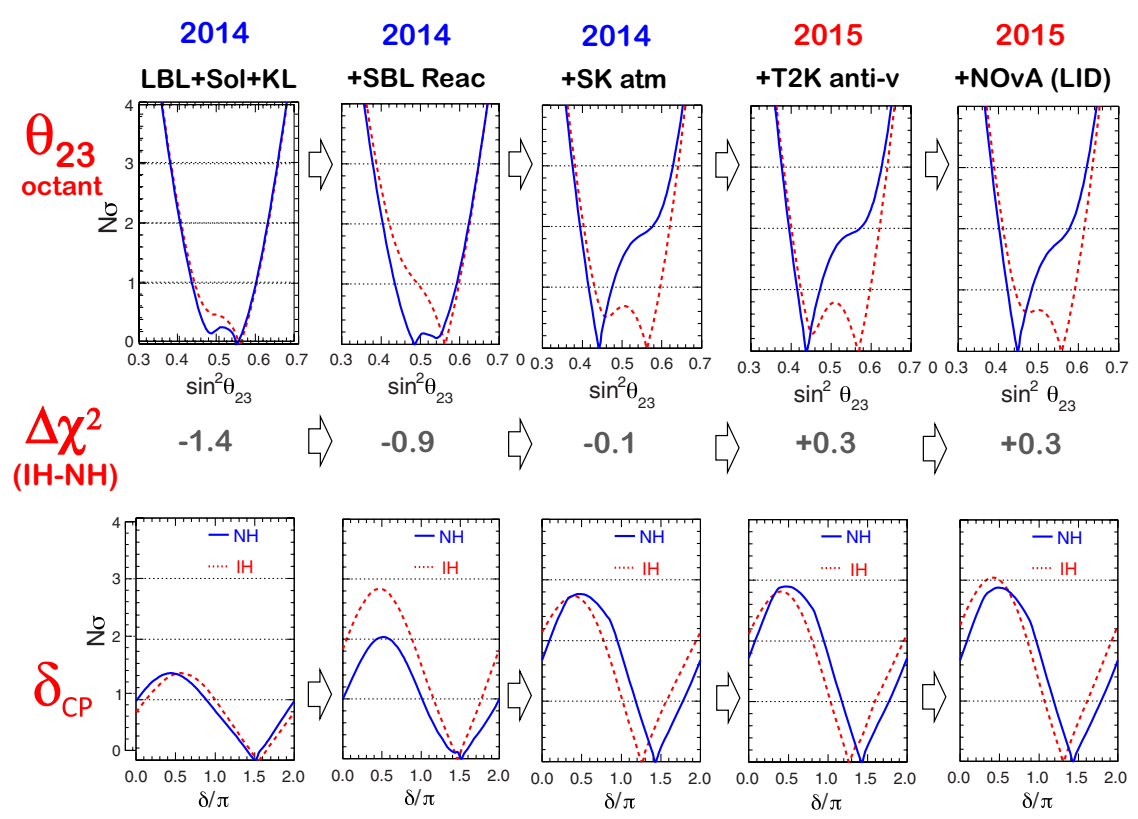

Figure 1. Global neutrino data analysis, with preliminary updates with respect to [3]. Upper panels: Contraints on $\sin ^{2} \theta_{23}$ in terms of $N_{\sigma}=\sqrt{\Delta \chi^{2}}$ for increasingly rich data sets. Lower panels: Corresponding constraints on the CP-violating phase $\delta$. Middle row: Corresponding values of the $\Delta \chi^{2}$ for hierarchy difference (IH vs NH).

to spectral shape systematics which may hinder, e.g., mass hierarchy signatures. We shall first discuss these issues in the relatively simple context of medium-baseline reactor projects, and then in connection with prospective atmospheric neutrino experiments in ice (or water).

\section{Global $3 v$ oscillation data analysis: Status of unknowns}

Our latest published global $v$ data analysis, presented in terms of $\chi^{2}$ best-fit values and allowed regions, was reported in [3]. In the context of unknown oscillation parameters, we illustrate here preliminary updated results including short-baseline (SBL) reactor $v$ data from the Neutrino 2014 Conference [4], as well as recent long-baseline (LBL) accelerator data from the T2K ( $\bar{v}$ run) and NOvA experiments in the $v_{\mu} \rightarrow v_{e}$ appearance channel, as appeared in 2015 [5]. A more complete analysis, including recent atmospheric data from Super-Kamiokande and IceCube [6], will be reported elsewhere [7].

Figure 1 shows the values of $\Delta \chi^{2}$ or, equivalently, $N_{\sigma}=\sqrt{\Delta \chi^{2}}$ for three unknown parameters: the $\theta_{23}$ octant, the mass hierarchy, and the CP-violating phase $\delta$. From left to right, the first three panels refer to the combination of LBL, solar and KamLAND data, plus SBL reactor data, plus SK atmospheric data, all updated to 2014 with respect to [3]. The last two panels contain, in addition, 2015 data from T2K and then from NOvA in the default (so-called LID) analysis [5]. It can be seen that, at 90\% C.L. $(\sim 1.6 \sigma)$, and even in the most complete data set (right panels), it is not possible to assign a specific octant to $\theta_{23}$, nor to select a preferred hierarchy: both octants and hierarchies are allowed. On the other hand, an intriguing hint emerges in favor of $\delta \neq 0, \pi$ and, in particular, in favor of $\sin \delta<0$, with a best fit close to $\delta \simeq 3 \pi / 2$. This value corresponds to a case of "maximal CP violation" ( $\sin \delta \simeq-1$ ), while the opposite maximal case $(\sin \delta \simeq+1)$ is disfavored at $\simeq 3 \sigma$.

Time will tell if this interesting hint for leptonic $\mathrm{CP}$ violation will be confirmed (or not) by future LBL data [5]. In the meantime, the mass hierarchy and $\theta_{23}$ octants might remain undetermined. The 

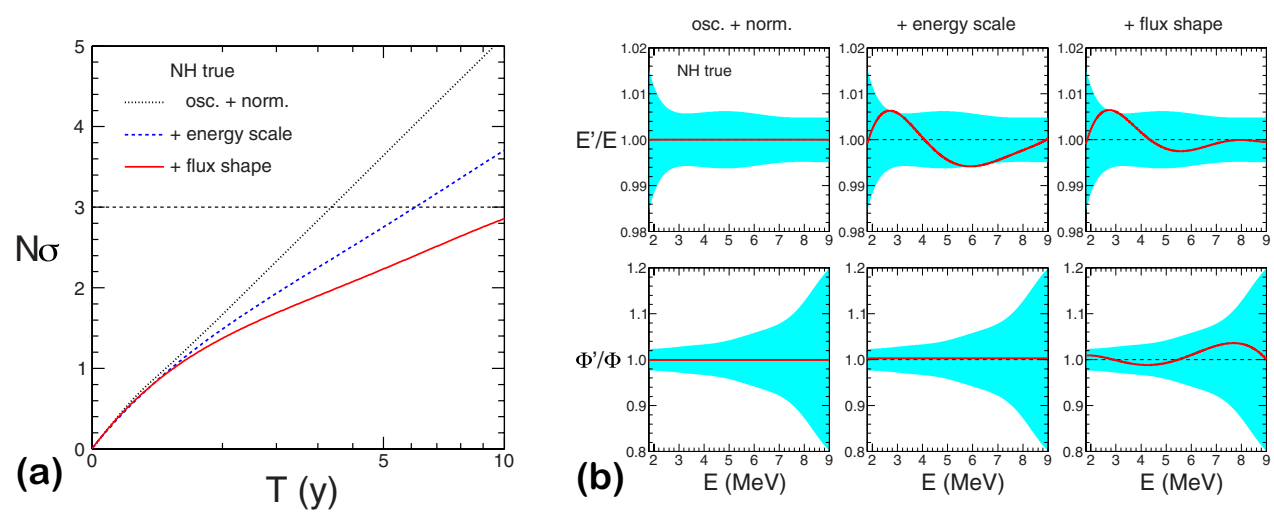

Figure 2. JUNO prospective data analysis for true NH (from [8]). Panel (a): Rejection power for the wrong IH, in terms of $N_{\sigma}$ as a function of time $T$ (with tics scaling as $\sqrt{T}$ ). From top to bottom, the curves include: oscillation parameters and flux normalization errors (osc.+norm), plus energy scale uncertainties $E^{\prime} / E$ (+energy scale), plus flux shape uncertainties $\Phi^{\prime} / \Phi$ (+ flux shape). Panel (b): Corresponding best-fits of the $E^{\prime} / E$ and $\Phi^{\prime} / \Phi$ energy profiles for prospective 5-yr data-taking, overlaid to the current $\pm 1 \sigma$ error bands (in blue). The case of true IH (not shown, see [8]) leads to similar results.

JUNO (reactor) and PINGU (atmospheric) neutrino projects have the potential to address the mass hierarchy issue (and PINGU also the $\theta_{23}$ octant) with future high-statistics measurements which, however, will present new challenges for global $v$ data analyses, as shown in the next sections.

\section{JUNO and systematics of one-dimensional neutrino spectra}

Unoscillated reactor neutrino spectra $\Phi(E)$, which depend only on one variable (the $v$ energy $E$ ), were thought to be accurately known at a few \% level until recently. However, a "bump" structure at $\sim 5 \mathrm{MeV}$ in prompt energy was discovered in the three independent experiments RENO, Daya Bay and Double Chooz [4], leading to $O(10 \%)$ local variations with respect to previous expectations, likely due to poorly known nuclear reaction spectral components. Conservatively, one should thus consider the possibility that the reactor spectra may be affected by continuous "functional" uncertainties on both the energy scale (spectrum $x$-axis, $E \rightarrow E^{\prime}(E)$ ), and the flux shape (spectrum $y$-axis, $\Phi(E) \rightarrow \Phi^{\prime}(E)$ ), especially in future experiments which will measure the oscillated spectra with high statistics and very good resolution, such as in the JUNO (China) and RENO-50 (South Korea) projects [4], which aim at determining the neutrino mass hierarchy via precision oscillometry.

In [8] we systematically performed the exercise of letting both ratios $E^{\prime} / E$ and $\Phi^{\prime} / \Phi$ vary smoothly, within their currently allowed error bands, in a JUNO-like experimental setting. Representative results for the mass hierarchy discrimination are shown in Fig. 2. In the left panel (a), the dotted black curve shows the increase of the mass hierarchy sensitivity in time, assuming solely the systematic uncertainties related to the oscillation parameters and to the reactor flux and geoneutrino background normalizations. Such curve basically scales as $\sqrt{T}$, showing that such systematics do not spoil significantly the intrinsic statistical sensitivity. However, including first the energy-scale errors and then the flux-shape uncertainties (as smooth variations of $E^{\prime} / E$ and $\Phi^{\prime} / \Phi$ from unity, respectively) the sensitivity is significantly degraded, and can eventually be $<3 \sigma$ even after 10 years of data taking. Panel (b) of the same figure shows the best-fit functional forms of $E^{\prime} / E$ and $\Phi^{\prime} / \Phi$, which are well within the colored $1 \sigma$ error bands. By halving such error bands, e.g., via proper calibrations, one could reach a sensitivity $>3 \sigma$ in a reasonable 5-yr time (not shown, see [8] for details). 

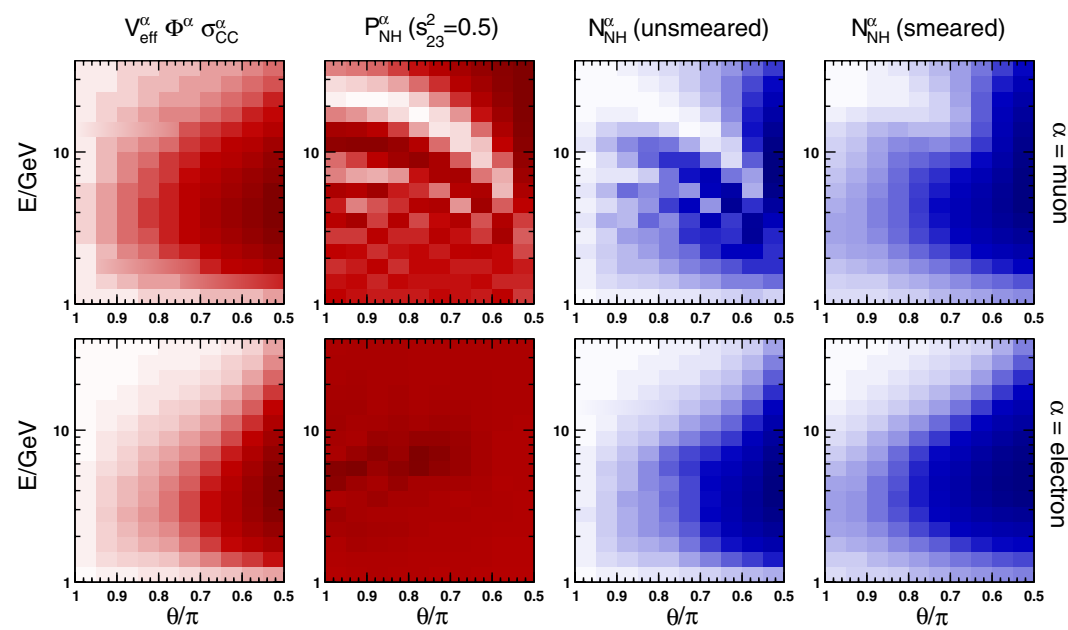
Unoscillated kernel

\section{Oscillation factor}

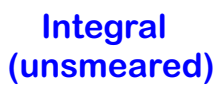

\section{Integral (smeared)}

Figure 3. Breakdown of the main ingredients of muon (upper panels) and electron (lower panels) spectra in PINGU (taken from [9]), in an arbitrary color scale ranging from white (null event rate) to dark colors for increasing event rates (with pixels representing data bins). From left to right: Unoscillated kernel (flux times target volume times cross-section); flavor oscillation factors for $\sin ^{2} \theta_{23}=\pi / 4$; resulting integral spectrum for perfect energy-angle resolutions (unsmeared) and finally for realistic resolutions (smeared). The final smeared spectra for NH (this figure) and for IH (not shown) would not be distinguishable by eye.

In conclusion, even for a relatively simple one-dimensional spectrum (such as $\Phi(E)$ at reactors), shape systematics may play a relevant role. The next section will deal with the more challenging case of two-dimensional event spectra in energy and angle.

\section{PINGU (ORCA) and systematics of two-dimensional neutrino spectra}

In this Workshop, extensive discussions have been focussed on future large-volume, high-statistics atmospheric neutrino experiments such as PINGU [6] and ORCA [10]. Here we report some results from our recent analysis of prospective data and sensitivities to the mass hierarchy and to $\theta_{23}$ in a PINGU-like experimental setting [9]. (Qualitatively, our results also apply to an ORCA-like setting.)

PINGU will measure the incoming energy and direction of muons and electrons (but with no lepton charge discrimination), which represent proxies for the unknown incoming energy $E$ and zenith angle direction $\theta$ of the parent neutrinos. The observable two-dimensional (energy-angle) spectra of $\mu$-like and $e$-like events are obtained as convolutions of several ingredients related to the source (atmospheric neutrino fluxes), to flavor change in propagation (oscillations), to the interaction process (cross section and target volume), and to detection characteristics (energy and angular resolutions).

Figure 3 shows how these ingredients determine the final spectral shapes. The convolution of an unoscillated kernel with oscillation factors, and then with energy-angle resolution functions, leads to observable spectra which have very broad structures and, in particular, do not display at a glance the oscillation patterns which encode the mass hierarchy signatures (see [9] for further details). However, the high statistics reachable by PINGU in a few years may be sufficient to discriminate the hierarchy via spectral analyses, provided that the sensitivity is not spoiled by systematic effects, which are unavoidably associated with any of the above ingredients. In this workshop, particular attention has been paid to uncertainties related to input atmospheric neutrino fluxes and neutrino cross sections [11], 

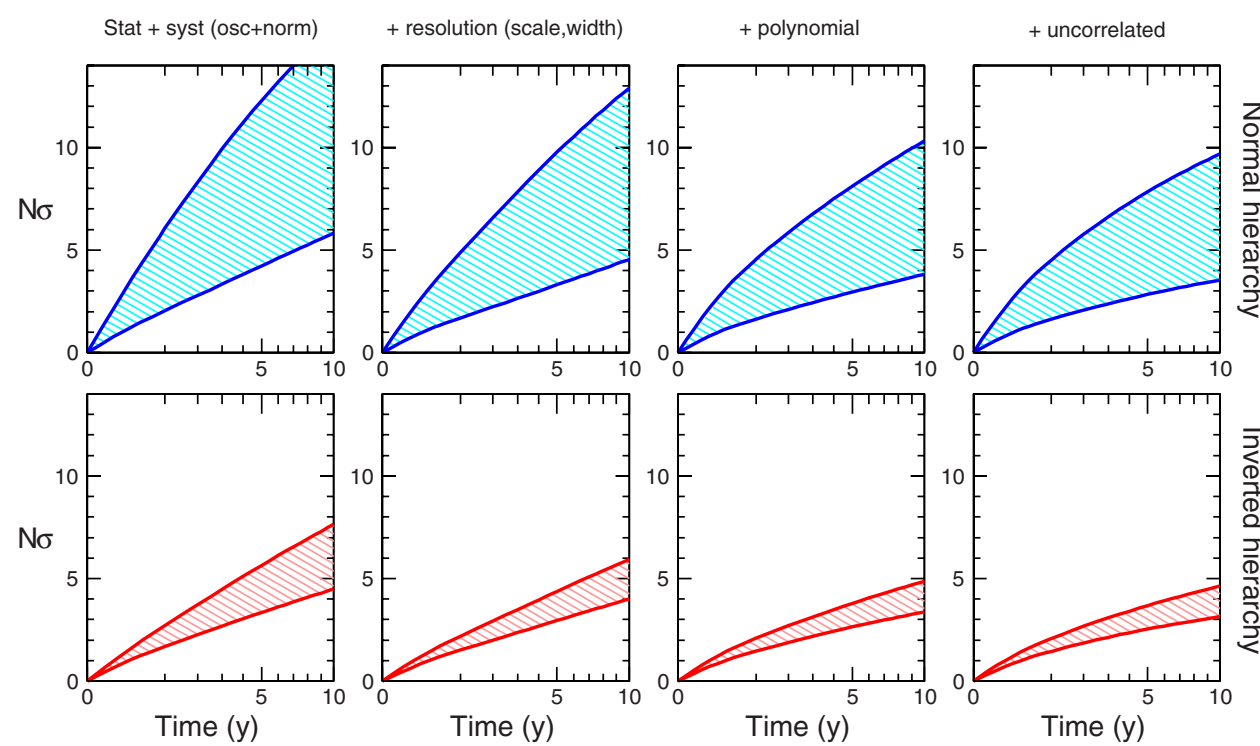

Figure 4. PINGU sensitivity to the hierarchy $\left(N_{\sigma}\right)$, for either true $\mathrm{NH}$ (top panels) or true IH (bottom panels), as a function of the live time $T$ in years. The abscissa is scaled as $\sqrt{T}$, so that the sensitivity bands would grow linearly for purely statistical errors. From left to right, the fit includes the following systematic errors: oscillation and normalization uncertainties, energy scale and resolution width errors, polynomial shape systematics (with up to quartic terms) at the $1.5 \%$ level, and uncorrelated systematics at the $1.5 \%$ level. See [9] for details.

but there are also other systematics related to the oscillating factors, to the energy-angle resolution functions, and to the effective target volume as a function of energy [9].

In [9] we have performed a thorough analysis of the effects of several systematic errors, both correlated and uncorrelated within bins, and with both known and uncertain energy-angle shape, in the context of PINGU. Figure 4 shows the main results of such work, in terms of sensitivity bands as a function of time for the cases of true $\mathrm{NH}$ (top) and true IH (bottom). The width of the bands is determined by the relatively large error on the $\theta_{23}$ mixing angle, which we take in the range $\sin ^{2} \theta_{23} \in[0.4,0.6]$. From left to right, the fit includes the following systematic errors: oscillation and normalization uncertainties, energy scale and resolution width errors, polynomial shape systematics (with up to quartic terms), and uncorrelated systematics. The last two error sources are kept at the default level of $1.5 \%$ (see [9] for a detailed definition of such uncertainties). Note that polynomial shape systematics are meant to parametrize smooth deviations from the expected shapes, due to a variety of energy-angle spectral uncertainties (atmospheric fluxes, differential cross sections, detector efficiencies, etc.) Including only normalization and systematic errors, the sensitivity $N_{\sigma}$ grows almost linearly with $T$, i.e., the experiment is not limited by these systematics, even after 10 years of data taking. However, the progressive inclusion of correlated shape systematics, both "known" (resolution scale and widths) and "unknown" (ad hoc polynomial deviations), and eventually of uncorrelated shape systematics, provide an overall decrease in the 5-year (10-year) PINGU sensitivity by up to $\sim 35 \%(\sim 40 \%)$. In particular, in the worst case of true $\mathrm{IH}$, a $3 \sigma$ discrimination of the hierarchy might take $\sim 10 \mathrm{yr}$.

However, it is possible to improve upon this situation by a better control of spectral shape systematics, which appears as a feasible goal by optimizing the PINGU configuration, and by studying in greater detail the effects of atmospheric flux and cross-section uncertainties [11]. As shown in [9], a reduction of the systematic errors in Fig. 4 by a factor of about two would definitely make it possible to discriminate the hierarchy at $>3 \sigma$ in less than 10 years in the worst case. Further benefits might come from a reduction of the $\theta_{23}$ uncertainty (see [9]). 


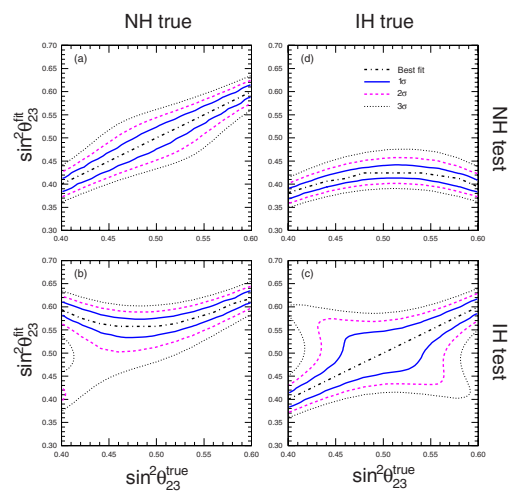

Figure 5. Fitted value $\sin ^{2} \theta_{23}^{\text {fit }}$ (at 1,2 and $3 \sigma$ ) versus the true value $\sin ^{2} \theta_{23}^{\text {true }}$, for the four possible cases where the test hierarchy (i.e., the one assumed in the fit) is either the true or the wrong one: (a) $\mathrm{NH}=$ true, $\mathrm{NH}=$ test; (b) $\mathrm{NH}=$ true, $\mathrm{IH}=$ test; (c) $\mathrm{IH}=$ true, $\mathrm{IH}=$ test; (d) $\mathrm{IH}=$ true, $\mathrm{NH}=$ test.

Although PINGU itself will contribute to improve the accuracy on $\theta_{23}$, the interpretation of its prospective $\theta_{23}$ bounds depends, in turn, upon the mass hierarchy determination: until the $\mathrm{NH} / \mathrm{IH}$ issue it is not settled and remains ambiguous, the $\theta_{23}$ bounds in PINGU may be significantly biased. Figure 5 shows the subtle interplay between hierarchy discrimination and $\theta_{23}$, in the four cases where the test hierarchy is equal (or not) to the true hierarchy. The cases where there is an hierarchy mismatch clearly lead to biased results for the $\theta_{23}$ bounds; see [9] for a more extensive discussion.

In conclusion, in the context of the standard $3 v$ framework and of a global data analysis, we have illustrated the status of the unknown oscillation parameters: the $\theta_{23}$ octant, the mass hierarchy, and the possible CP phase $\delta$. We have then discussed the challenges related to spectral shape uncertainties in some future experiments, which aim at determining the mass hierarchy via one-dimensional event spectra (e.g., JUNO and RENO-50 with reactor neutrinos) or two-dimensional event spectra (e.g., PINGU and ORCA with atmospheric neutrinos). Improvements in our understanding and control of spectral shape systematics are needed to safely reach $>3 \sigma$ sensitivities for the $\mathrm{NH} / \mathrm{IH}$ discrimination.

E.L. thanks the VLVNT 2015 organizers for kind hospitality and support. This work is supported by INFN and MIUR through the "Theoretical Astroparticle Physics" project.

\section{References}

[1] www.nobelprize.org/nobel_prizes/physics/laureates/2015/

[2] K.A. Olive et al. (Particle Data Group), Chin. Phys. C 38, 090001 (2014)

[3] F. Capozzi et al., Phys. Rev. D 89, 093018 (2014) [arXiv:1312.2878 [hep-ph] ]

[4] Neutrino 2014, XXVI International Conf. on v Physics and Astrophysics, neutrino2014. $\mathrm{bu} . \mathrm{edu} /$

[5] A. Weber, talk at this Workshop

[6] J.-P. Yanez, talk at this Workshop

[7] F. Capozzi, E. Lisi, A. Marrone, D. Montanino, and A. Palazzo (2016), in preparation.

[8] F. Capozzi et al., Phys. Rev. D 92, 093011 (2015) [arXiv:1508.01392 [hep-ph]]

[9] F. Capozzi et al., Phys. Rev. D 91, 073011 (2015) [arXiv:1503.01999 [hep-ph] ]

[10] D. Samtleben, talk at this Workshop

[11] J. Nieves, J. Sandroos, A. Gazizov, talks at this Workshop 